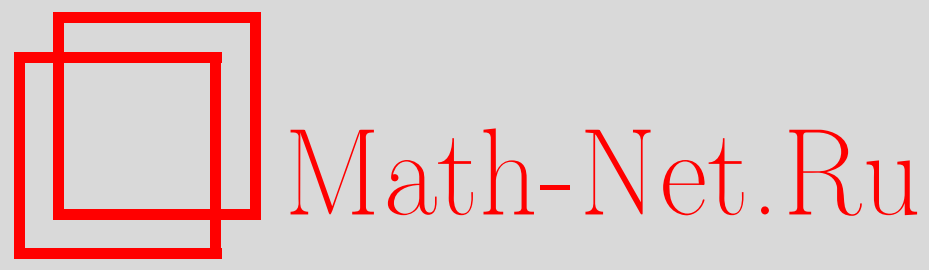

И. А. Пушкарь, Многомерное обобщение теоремы Ильяшенко об абелевых интегралах, Функи. анализ и его прил., 1997, том 31, выпуск 2, 34-44

DOI: https://doi.org/10.4213/faa461

Использование Общероссийского математического портала MathNet.Ru подразумевает, что вы прочитали и согласны с пользовательским соглашением

http://www . mathnet.ru/rus/agreement

Параметры загрузки:

IP : 3.82 .47 .9

26 апреля 2023 г., $17: 20: 56$

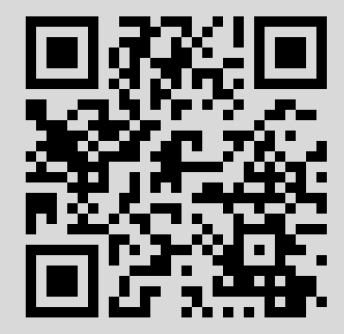




\title{
Многомерное обобщение теоремы Ильяшенко об абелевых интегралах
}

\author{
(с) 1997. И. А. ПушкАРь
}

\section{Введение}

Как известно [1], циклы, рождающиеся при полиномиальном возмущении полиномиального гамильтонова векторного поля на плоскости, связаны с нулями абелевых интегралов, рассматриваемых как функции параметров. В этой связи Ю. С. Ильяшенко заинтересовался нулями абелевых интегралов и доказал следующую теорему [2].

ТЕОремА. Пусть $P(x, y)$ - вещественный многочлен степени $n>2$ от двух переменных, у которого все комплексные критические точки морсовские, а все критические значения различны. Пусть старшая однородная часть многочлена невырожденна. Пусть $P=0$ - некритическая поверхность уровня, $\alpha=A d x+B d y$ - полиномиальная 1-форма и $A(x, y) u$ $B(x, y)$ - многочлень степени не выше $n-1$. Пусть $\gamma(0)$ - исчезаюший иикл на комплексной кривой $P=0$, лежащий на вещественной плоскости, и $\gamma(c) \subset(P=c)$ - непрерьвная деформаиия этого иикла. Пусть при достаточно мальх значениях параметра с справедливо тождество

$$
\int_{\gamma(c)} \alpha \equiv 0 \text {. }
$$

Тогда а-точная форма.

В настоящей работе предлагается другой, отличный от авторского, подход к теореме Ильяшенко. Наш подход использует соображения теории особенностей и алгебраической геометрии. Он позволяет обобщить теорему Ильяшенко в следующих направлениях. Во-первых, снимается ограничение на цикл $\gamma(0)$ - в нашей теореме он не обязательно исчезающий. Во-вторых, утверждение переносится на случай многих переменных. А именно доказывается следующая

ОСНОВНАЯ ТЕОРЕмА. Пусть $P\left(x_{1}, \ldots, x_{k}\right)$ - вешественный многочлен степени $n>k$, у которого все комплекснье критические точки морсовские, а все критические значения различны. Пусть старшая однородная часть многочлена невырожденна. Пусть $P=0$ - некритическая поверхность уровня, а $\alpha$ - полиномиальная $(k-1)$-форма, коэффициенть которой являются многочленами степени не выле $n-k+1$. Пусть $\gamma(0)$ - снабженная любой ориентачией компактная компонента связности вещественной

* Работа выполнена при частичной поддержке Российского фонда фундаментальных исследований (грант 95-01-01258) и ИНТАС (грант 93570). 
поверхности $P=0$ и $\gamma(c) \subset(P=c)$ - ее непрерьвная деформаиия. Пусть при достаточно мальх значениях параметра с справедливо тождество

$$
\int_{\gamma(c)} \alpha \equiv 0 .
$$

Тогда $а$ - точная форма.

В двумерном случае наше доказательство не только проще первоначального, но и позволяет получить новую информацию о возможном расположении предельных циклов полиномиального векторного поля на плоскости.

СЛЕДСТВИЕ. Пусть $H(x, y)$ - произвольный многочлен степени $n>2$, $m=(n-1) n / 2-1 u \gamma_{1}, \ldots, \gamma_{m}$ - произвольные компактные компоненты связности неособых линий уровня $H=c_{1}, \ldots, H=c_{m}$ многочлена Н. Для любого $\varepsilon>0$ существует полиномиальное векторное поле степени $n-1$ на плоскости, у которого найдутся предельнье иикльи $\tilde{\gamma}_{1}, \ldots, \tilde{\gamma}_{m}$, отстояшие от соответствуюших кривых $\gamma_{1}, \ldots, \gamma_{m}$ на расстояние, не превосходящеe $\varepsilon$.

В работе [2] было доказано аналогичное следствие, однако в ней приходилось налагать ограничения на расположение кривых $\gamma_{1}, \ldots, \gamma_{m}$.

Отметим, что в [3] найдено другое обобщение части результатов работы [2].

Я признательна Ю. С. Ильяшенко за постановку задачи и внимание к работе, а также А. Г. Хованскому за полезные обсуждения.

\section{§1. Топология гиперповерхности}

В этом параграфе приводятся известные результаты о топологии общей комплексной аффинной гиперповерхности фиксированной степени и ее проективного замыкания.

Пусть $P\left(x_{1}, \ldots, x_{k}\right)$ - морсовский многочлен в $\mathbb{C}^{k}$ (т. е. многочлен, все критические точки которого невырожденны и все критические значения различны) степени $n$. Пусть его старшая однородная часть $P_{n}$ невырожденна (т. е. однородный многочлен $P_{n}$ не имеет критических точек, за исключением точки 0 ). Пусть число 0 не является критическим значением многочлена $P$. Обозначим через $U \subset \mathbb{C}^{k}$ аффинную поверхность уровня $P=0$, через $X \subset \mathbb{C P}^{k}-$ компактификацию поверхности $U$ в $\mathbb{C} P^{k}$ и через $S=X \backslash U$ - пересечение $X$ с бесконечно удаленной гиперплоскостью.

Рассмотрим вложения $i: U \rightarrow X$ и $j: S \rightarrow X$. Им соответствует отображение групп гомологий

$$
i_{*}: H_{k-1}(U) \rightarrow H_{k-1}(X), \quad j_{*}: H_{k-1}(S) \rightarrow H_{k-1}(X) .
$$

Цикл $\gamma \in H_{k-1}(X)$, лежащий в образе отображения $i_{*}$, называется примитивньлм ииклом.

Хорошо известно следующее

РАЗЛОЖЕНИЕ ЛЕФШЕЦА. Отображение $j_{*}: H_{k-1}(S) \rightarrow H_{k-1}(X)$ является вложением. Группа гомологий средней размерности многообразия $X$ 
является прямой суммой примитивных гомологий и гомологий «бесконечно удаленного» сечения $S$. Другими словами, справедливо следующее разложение:

$$
H_{k-1}(X)=\operatorname{Im} i_{*} \oplus \operatorname{Im} j_{*} .
$$

В группах гомологий средней размерности $H_{k-1}(U)$ и $H_{k-1}(X)$ индекс пересечения определяет билинейные формы. По двойственности Пуанкаре форма пересечения на $H_{k-1}(X)$ невырожденна, так как $X$ неособо и компактно. Но форма пересечения на $H_{k-1}(U)$ может иметь нулевое подпространство, которое мы обозначим через $L$.

ЛеммА. Цикль из $H_{k-1}(U)$, имеюшие нулевое пересечение с любими ииклами из $H_{k-1}(U)$, и только они при вложении $U$ в $X$ становятся гомологичнылми нулю. Другими словами, $\operatorname{Ker} i_{*}=L$.

ДокаЗАтЕльство. 1. Если $\gamma \in \operatorname{Ker} i_{*}$, то в $X$ цикл $\gamma$ гомологичен нулю и индекс пересечения его с любым циклом из $X$ (а значит, и из $U$ ) равен нулю, т. е. $\gamma \in L$.

2. Если $i_{*}(\gamma) \neq 0$, то по двойственности Пуанкаре в $X$ найдется цикл $\gamma^{\prime}$, такой, что $\left(\gamma, \gamma^{\prime}\right) \neq 0$. По разложению Лефшеца $\gamma^{\prime}=\gamma_{1}+\gamma_{2}$, где $\gamma_{1} \subset U$, $\gamma_{2} \subset S$. Поэтому $\left(\gamma, \gamma^{\prime}\right)=\left(\gamma, \gamma_{1}\right)+\left(\gamma, \gamma_{2}\right)$.

Далее, $\left(\gamma, \gamma_{2}\right)=0$, так как циклы $\gamma$ и $\gamma_{2}$ не пересекаются. Если $\gamma \in L$, то $\left(\gamma, \gamma_{1}\right)=0$. Противоречие доказывает нужное утверждение.

Вернемся к аффинной неособой гиперповерхности $U$, определенной в $\mathbb{C}^{k}$ полиномиальным уравнением $P=0$ с невырожденной старшей однородной частью. Хорошо известна следующая

ТЕОРЕМА О ГОМОТОПИЧЕСКОМ ТИПЕ ГИПЕРПОВЕРХНОСТИ. Гиперповерxность $U$ гомотопически эквивалентна букету из $(n-1)^{k}$ сфер размерности $k-1$. Группа гомологий средней размерности гиперповерхности $U$ порождена исчезающими ииклами.

\section{$\S 2$. Разрешение особенностей дискриминанта}

Рассмотрим пространство многочленов $P$ с фиксированной невырожденной старшей однородной частью $P_{n}, P=P_{n}+P_{a}$, где $a=\left(a_{1}, \ldots, a_{N}\right)$ - остальные коэффициенты многочлена, называемые в дальнейшем параметрами.

Дискриминантная поверхность $\Sigma$ лежит в пространстве параметров и определена следуюшим условием: $a \in \Sigma$, если и только если гиперповерхность $P_{n}+$ $P_{a}=0$ имеет особую точку.

Поверхность $\Sigma$, вообще говоря, особая. Построим явное разрешение особенностей этой поверхности. Аналогичное построение, связанное с ростком дискриминанта, можно найти в $[4$, с. 56$]$.

Определим $\widetilde{\Sigma}$ как множество пар $(x, P)$, где $x \in \mathbb{C}^{k}$ и многочлен $P=P_{n}+P_{a}$ обрашается в нуль в точке $x$, причем $x$ является критической точкой этого многочлена. Множество $\widetilde{\Sigma}$ можно рассматривать как аффинное алгебраическое многообразие. Рассмотрим проекцию $\pi$ алгебраического многообразия $\widetilde{\Sigma}$ в пространство параметров, сопоставляющее точке $(x, P)$, где $P=P_{n}+P_{a}$, параметр $a$. 
ТЕоремА. (1) При $n \geqslant 2$ алгебраическое многообразие $\widetilde{\Sigma}$ бирегулярно эквивалентно линейному пространству $\mathbb{C}^{N-1}$.

(2) При $n \geqslant 2$ проекчия $\pi$ отображает $\widetilde{\Sigma}$ на $\Sigma$. Это отображение собственно и является бирегулярной эквивалентностью вне собственньх алгебраических подмножеств алгебраических многообразий $\widetilde{\Sigma} u \Sigma$.

ДокаЗАтельство. (1) Обозначим через $P^{0}$ пространство многочленов с нулевым свободным членом, нулевой линейной частью и старшей однородной частью $P_{n}$. Пространство $P^{0}$ является комплексным линейным пространством размерности $N-(k+1)$. Точка $\left(x_{0}, P\right)$ принадлежит $\widetilde{\Sigma}$, если и только если многочлен $P\left(x+x_{0}\right)$ лежит в пространстве $P^{0}$. Это описание и дает искомую бирегулярную эквивалентность пространств $\mathbb{C}^{k} \times \mathbb{C}^{N-k+1}=\mathbb{C}^{N-1}$ и $\tilde{\Sigma}$.

(2) Рассмотрим подмножество $\Sigma_{n}$ дискриминантной поверхности $\Sigma$, состоящее из многочленов $P$, имеющих ровно одну критическую точку на поверхности нулевого уровня $P=0$, для которых эта единственная особая точка невырожденна. Несложно показать, что: (а) подмножество $\Sigma_{n}$ является гладким многообразием, (b) дополнение $\Sigma_{0}=\Sigma \backslash \Sigma_{n}$ является алгебраическим подмножеством в $\Sigma$, имеющим меньшую размерность, (с) отображение $\pi$ устанавливает бирегулярную эквивалентность множества $\widetilde{\Sigma}_{n}=\pi^{-1}\left(\Sigma_{n}\right)$ и множества $\Sigma_{n}$.

\section{§3. Группы монодромии}

Поверхности уровня в $\mathbb{C}^{k}$ многочлена $P$ образуют расслоение над дополнением к дискриминантной поверхности $\Sigma$ в пространстве коэффициентов. С этим расслоением связаны линейные расслоения с той же базой, слоями которых являются пространства гомологий пространства $U$ всевозможных размерностей. Фундаментальная группа базы имеет представления в этих пространствах, причем ее образами являются группы монодромии гомологий соответствующих размерностей.

ЛЕмМА. В гомологиях средней размерности аффинной поверхности группа монодромии действует транзитивно на исчезаюших ииклах, точнее, любой исчезаюший иикл можно перевести элементом группь монодромии в любой другой исчезающий иикл ( с точностью до знака).

ДокАЗАТЕльство. Прообраз $\widetilde{\Sigma}_{0}$ множества $\Sigma_{0}$ при разрешении особенностей $\pi: \widetilde{\Sigma} \rightarrow \Sigma$ является алгебраическим подмногообразием в $\widetilde{\Sigma}$. Множество $\widetilde{\Sigma} \backslash \widetilde{\Sigma}_{0}$ связно, так как оно бирегулярно эквивалентно дополнению пространства $\mathbb{C}^{N-1}$ до алгебраического подмножества. Поэтому множество $\Sigma_{n}=\Sigma \backslash \Sigma_{0}$ связно.

Укажем элемент фундаментальной группы пространства $\mathbb{C}^{N} \backslash \Sigma$, переводящий исчезающий цикл $\gamma_{1}$ в исчезающий цикл $\pm \gamma_{2}$. Пусть цикл $\gamma_{i}$ исчезает вдоль пути $\alpha_{i}$ в точке $d_{i} \in \Sigma_{n}$. Можно считать, что путь $\alpha_{i}$ пересекает $\Sigma$ лишь в конечной точке $d_{i}$. Точки $d_{1}$ и $d_{2}$ можно соединить путем $\beta$, лежащим в $\Sigma_{n}$. Рассмотрим путь $\tau$, близкий к пути $\alpha_{1} \beta \alpha_{2}^{-1}$, но не имеющий с $\Sigma$ общих точек. Путь $\tau$ переводит цикл $\gamma_{1}$ в цикл $\pm \gamma_{2}$.

ЗАмЕчАниЕ. Аналогичное рассуждение, связанное с локальной группой монодромии, можно найти в [4, с. 57-58]. 
ТЕОРема. При действии группь монодромии в гомологиях средней размерности аффинной гиперповерхности $U$ элементы пространства $L$ u только они остаются поточечно неподвижными. Если же чикл не принадлежит пространству $L$, то его образы при действии группы монодромии порождают все линейное пространство $H_{k-1}(U)$.

ДокаЗАТЕльство. Бесконечно удаленное сечение $S$ является неособым подмногообразием гиперповерхности $X$, так как старшая однородная часть $P_{n}$ уравнения $P=0$ невырожденна. Пусть $\gamma \in L$. Рассмотрим отрезок точной последовательности Лере (см. [5])

$$
H_{k-2}(S) \stackrel{\delta}{\rightarrow} H_{k-1}(U) \stackrel{i_{*}}{\rightarrow} H_{k-1}(X) .
$$

Так как эта последовательность точна, то $\operatorname{Ker} i_{*}=\operatorname{Im} \delta$. Значит, по лемме из $\S 1 \gamma \in L$ тогда и только тогда когда $\gamma \in \operatorname{Im} \delta$. Ясно, что при действии группы монодромии циклы из пространства $\operatorname{Im} \delta$ остаются неподвижными.

Пусть $\gamma \notin L$. Тогда найдется цикл $\gamma^{\prime}$ из $U$, такой, что $\left(\gamma, \gamma^{\prime}\right) \neq 0$. Значит, найдется исчезающий цикл $\gamma_{i}$, для которого $\left(\gamma, \gamma_{i}\right) \neq 0$, так как исчезающие циклы порождают группу $H_{k-1}(U)$ (см. $\left.\S 1\right)$. По теореме Пикара-Лефшеца (см. $[4$, c. 23]) мы можем так подействовать на $\gamma$ элементом группы монодромии, что $\gamma$ перейдет в $\gamma \pm\left(\gamma, \gamma_{i}\right) \gamma_{i}$. То есть один из исчезающих циклов $\gamma_{i}$ порождается образами элемента $\gamma$. Поэтому, согласно предыдущей лемме, любой из исчезающих циклов порождается образами элемента $\gamma$. Доказательство закончено, так как исчезающие циклы порождают все пространство $H_{k-1}(U)$ (см. $\left.\S 1\right)$.

СлЕДСтвиЕ. Если степень $n$ гиперповерхности $X$, равнал $\operatorname{deg} P$, больше 2 , то никакой исчезающий иикл не принадлежит пространству $L$.

ДокАЗАТЕльство. Действительно, при $n>2$ число $(n-1)^{k}$ исчезающих циклов больше единицы. По предыдущей лемме исчезающие циклы не остаются неподвижными при действии монодромии и, согласно теореме, не лежат в пространстве $L$.

\section{§4. Локальная теорема Зарисского и группа монодромии}

Пусть $A \subset \mathbb{C}^{n}$ - аффинная алгебраическая гиперповерхность и $l$ - достаточно общая комплексная прямая, лежащая в $\mathbb{C}^{n}$.

ГЛОБАЛЬНАЯ ТЕОРЕМА ЗАРИССКОГО. Отображение вложения

$$
i_{*}: \pi_{1}(l \backslash A) \rightarrow \pi_{1}\left(\mathbb{C}^{n} \backslash A\right)
$$

является отображением «на».

Известен следующий локальный вариант теоремы Зарисского.

Рассмотрим аналитическую гиперплоскость $A$ в достаточно малом шаре $B$ вокруг точки 0 . Пусть $l$ - прямая, проходящая через точку 0, и $l_{\varepsilon}$ - семейство параллельных ей прямых $\left(l_{0}=l\right)$.

ЛОКАЛЬНАЯ ТЕОРЕМА ЗАРИССКОГО. Для достаточно общего направления $l$ и для почти всех достаточно мальх $\varepsilon$ отображение

$$
\pi_{1}\left(\left(l_{\varepsilon} \cap B\right) \backslash A\right) \rightarrow \pi_{1}(B \backslash A)
$$


В локальной теореме Зарисского имеются в виду следующие условия общности:

1. Общность направления $l$. Прямая $l$ называется общей для ростка гиперповерхности $F=0$, если младшая однородная часть $F_{\mu}$ функции $F=$ $F_{\mu}+F_{\mu+1}+\ldots$ на прямой $l$ не обращается тождественно в нуль.

2. Условие общности прямой $l_{\varepsilon}$. Прямая $l_{\varepsilon}$ должна пересекать росток гиперповерхности $F=0$ лишь в его неособых точках и трансверсально. При этом $\varepsilon$ должно быть столь малым, чтобы число точек пересечения в шаре $B$ равнялось $\mu$.

Рассмотрим росток дискриминантной поверхности $\Sigma$ многочленов $P=$ $P_{n}+P_{a}$ в окрестности точки $a=0$. Пусть $a_{N}=c-$ свободный член многочлена $P$. Младшая однородная часть неприводимого уравнения ростка дискриминантной поверхности в нуле пропорциональна $c^{\mu}$, где $\mu=(n-1)^{k}$. Доказательство этого факта можно найти в книге [4].

Пусть $P=P_{n}+P_{a}$ - морсовский многочлен. Рассмотрим прямую $P-c$, где $c \in \mathbb{C}$ - комплексный параметр, в пространстве таких многочленов. Множество $K=\left\{c_{i}\right\}$ критических значений многочлена $P$ содержит ровно $\mu=(n-1)^{k}$ точек. Многочлены $P-c$, где $c \in \mathbb{C} \backslash K$, имеют невырожденную поверхность нулевого уровня.

ТЕорема 1. Вложение множества $\mathbb{C} \backslash K$ в дополнение $\kappa$ дискриминантной поверхности $\Sigma$, заданное формулой $c \mapsto P-c$, задает отображение фундаментальной әруппь $\pi_{1}(\mathbb{C} \backslash K)$ на фундаментальную әруппу $\pi_{1}\left(\mathbb{C}^{N} \backslash \Sigma\right)$.

ДокАЗАтЕльство. Теорема выводится из локальной теоремы Зарисского. Ясно, что при $\varepsilon \neq 0$ многочлены $P$ и $\varepsilon^{n} P(x / \varepsilon)$ принадлежат или не принадлежат поверхности $\Sigma$ одновременно. Преобразование $P \rightarrow \varepsilon^{n} P(x / \varepsilon)$ сводит глобальный вопрос о фундаментальной группе дополнения к $\Sigma$ к локальному, т. е. к вопросу о фундаментальной группе $B \backslash \Sigma$, где $B$ - малый шар вокруг точки 0 .

Действительно, прямая $P-c$ пересекает множество $\Sigma$ в $\mu$ точках $P-c_{i}$, где $c_{i} \in K$. При $\varepsilon \neq 0$ преобразованная прямая $\varepsilon^{n}(P(x / \varepsilon)-c)$ будет пересекать поверхность $\Sigma$ тоже в $\mu$ точках. Все эти точки при достаточно малом $\varepsilon$ будут лежать в фиксированном шаре $B$. Поэтому для преобразованной прямой $l_{\varepsilon}$ выполнено условие общности положения. Теперь теорема вытекает из локальной теоремы Зарисского.

Поверхности уровня $P=c$ образуют расслоение над $\mathbb{C} \backslash K$. Фундаментальная группа базы $\mathbb{C} \backslash K$ действует в пространстве гомологий каждой размерности.

Теорема 2. При действии группь монодромии в гомологиях средней размерности слоя $P-c=0$ расслоения с базой $\mathbb{C} \backslash K$ элементы из ядра $L$ формы пересечения и только они остаются поточечно неподвижньми. Если же иикл не принадлежит пространству L, то его образы при действии группь монодромии порождают все линейное пространство $H_{k-1}(U)$.

Теорема 2 непосредственно вытекает из теоремы 1 и из теоремы $\S 3$.

\section{§5. Следствия теории Ходжа-де Рама}

Нам понадобится следующий результат из теории гармонических форм на кэлеровых многообразиях: 
Теорема (Ходж-де Рам). Если голоморфная l-форма $\omega$ на кэлеровом компактном проективном многообразии $X, \operatorname{dim}_{\mathbb{C}} X=l$, задает нулевой класс l-мерных когомологий, то она тождественно равна 0 .

Из этой теоремы, используя разложение Лефшеца и вычеты Лере, можно вывести нужные нам теоремы 1 и 2, которые формулируются и доказываются в этом параграфе.

Теорема 1. Пусть $X$ - гладкое компактное проективное многообразие, $S$ - его общее гиперплоское сечение и $U=X \backslash S$. Пусть для голоморфной формь $\omega$ старшей степени на $X$ и любого иикла $\gamma \in H_{l}(U)$, где $l=\operatorname{dim}_{\mathbb{C}} X$, выполнено равенство

$$
\int_{\gamma} \omega=0
$$

Тогда $\omega \equiv 0$.

ДокаЗАтельство. 1. Пусть $\tau \in H_{l}(S)$. Тогда $\int_{\tau} \omega=0$. Действительно, форма $\omega$ при ограничении на гиперплоское сечение тождественно обращается в нуль, так как комплексная размерность гиперплоского сечения равна $l-1$ и на нем нет ненулевых голоморфных $l$-форм.

2. Для всякого цикла $\sigma \in H_{l}(X)$ выполнено равенство

$$
\int_{\sigma} \omega=0
$$

Действительно, согласно разложению Лефшеца (см. §1),

$$
\sigma=\tau+\gamma,
$$

где $\tau \in H_{l}(S)$, а $\gamma \in H_{l}(U)$. Но интегралы формы $\omega$ по этим циклам равны нулю.

3. Итак, $\omega$ определяет нулевой класс $l$-мерных когомологий $X$. Следовательно, согласно теореме Ходжа-де Рама, $\omega \equiv 0$.

Теорема 2. Пусть $X$ - гладкое комплексное проективное многообразие, $\operatorname{dim}_{\mathbb{C}} X=l, S$ - его общее гиперплоское сечение и $U=X \backslash S$. Пусть $\omega$ есть l-форма, голоморфнал на $U$ и имеющая на $S$ полюс не выше первого порядка. Пусть для любого иикла $\gamma \in H_{l}(U)$

$$
\int_{\gamma} \omega=0
$$

Тогда $\omega \equiv 0$.

ДокАЗАТЕЛЬСтво. Форма $\omega$ по условию имеет полюс не выше первого порядка на гиперплоском сечении $S$. Поэтому на $S$ определена голоморфная форма-вычет $\omega_{l-1}$, такая, что для всякого цикла $\tau \in H_{l-1}(S)$ справедливо равенство

$$
2 \pi i \int_{\tau} \omega_{l-1}=\int_{\delta(\tau)} \omega_{l}
$$

где $\delta$ - граничный оператор Лере [5]. Однако по условию $\int_{\delta(\tau)} \omega=0$. 
Таким образом, интеграл формы $\omega_{l-1}$ по всякому циклу $\tau \in H_{l-1}(S)$ равен нулю. Форма $\omega_{l-1}$ - голоморфная форма на кэлеровом многообразии $S$. По теореме Ходжа-де Рама она тождественно равна нулю.

Итак, форма $\omega$ при сделанных предположениях не имеет полюса вдоль гиперплоского сечения $S$ и, следовательно, является голоморфной на многообразии $X$. По теореме $1 \omega \equiv 0$.

\section{§6. Исследование полюсов}

В пространстве $\mathbb{C}^{k}$ рассмотрим форму старшего порядка $\omega=Q d x_{1} \wedge \ldots$ $\wedge d x_{k}$, где $Q$ - многочлен. В неособых точках поверхности $P=0$ существует форма $\tau$, такая, что $\omega=\tau \wedge d P$. Ограничение формы $\tau$ на касательное пространство к поверхности $P=0$ определено корректно и обозначается символом $\omega / d P$. Предположим, что старшая однородная часть многочлена $P$ невырожденна и что 0 не является его критическим значением. В этих условиях справедлива

ТЕорема. Форма $\omega / d P$ мероморфна на проективной компактификаиии поверхности $P=0$. При этом она голоморфна в аффинной части поверхности, а на бесконечности имеет полюс порядка не больше, чем $\operatorname{deg} Q-\operatorname{deg} P+$ $k+1$. В частности, борма $\omega / d P$ голоморфна, если $\operatorname{deg} Q \leqslant \operatorname{deg} P-k-1, u$ имеет полюс не выише первого порядка, если $\operatorname{deg} Q \leqslant \operatorname{deg} P-k$.

Теорема доказывается прямым вычислением (ср. [6]).

\section{§7. Доказательство комплексного варианта основной теоремы}

Рассмотрим голоморфную $(k-1)$-форму $\alpha$ в $\mathbb{C}^{k}$.

Положим $I(c)=\int_{\gamma_{c}} \alpha$, где $\gamma_{c}$ - цикл, непрерывно зависящий от параметра $c$ и лежащий на поверхности $P\left(x_{1}, \ldots, x_{k}\right)=c$.

Лемма. (1) $I$ - росток функиии, аналитической на универсальной накрываюшей дополнения до множества критических значений многочлена $P$.

(2) $d I(c) / d c=\int_{\gamma_{c}} d \alpha / d P$.

ДокАЗАТЕльство. (1) следует из аналитической зависимости интеграла от параметра.

(2) См. теорему Лере-Гельфанда [4, с. 159].

Пусть $P$ - комплексный морсовский многочлен степени $n$ с невырожденной старшей частью и невырожденной поверхностью нулевого уровня.

Положим $d \alpha=\omega$. Рассмотрим функцию

$$
J(c)=\int_{\gamma_{c}} \frac{\omega}{d P} .
$$

ПРЕДЛОЖЕНИЕ. Пусть $\gamma_{0}$ - иикл на аффинной комплексной поверхности $P=0$, не гомологичный нулю на ее проективной компактификации. Пусть функиия $J(c)$ тождественно равна нулю при мальх изменениях параметра с. Тогда для любого иикла $\gamma$, лежащего на аффинной поверхности $P=0$,

$$
\int_{\gamma} \frac{\omega}{d P}=0
$$


ДокаЗАТЕльство. Рассмотрим аналитическое продолжение функции $J(c)$. По теореме 2 из $\S 4$ образы цикла $\gamma_{0}$ при действии группы монодромии порождают всю группу гомологий средней размерности аффинной поверхности $P=0$. Для любого цикла $M\left(\gamma_{0}\right)$, являющегося образом цикла $\gamma_{0}$ при действии группы монодромии $M, \int_{\gamma} \omega / d P=0$, так как аналитическое продолжение нулевой функции тождественно равно нулю.

КОМПЛЕКСНЫЙ ВАРИАНТ ОСНОВНОЙ ТЕОРЕМЫ. Пусть $P-$ комплексный морсовский многочлен с невырожденной старшей однородной частью $P_{n} u$ невырожденной поверхностью нулевого уровня. Пусть $\alpha$ - полиномиальная $(k-1)$-форма, коэффичиенты которой - многочлены степени не выше $n$ $k+1$. Пусть $(k-1)$-мерный цикл $\gamma_{0} \subset(P=0)$ не гомологичен нулю на проективной компактификачии этой поверхности и иикл $\gamma(c) \subset(P=c)$ непрерьвно зависит от малого параметра с. Тогда

$$
\int_{\gamma(c)} \alpha \equiv 0
$$

если и только если борма а точна.

ДокаЗАтЕльство. Рассмотрим функцию $J(c)=\partial I(c) / \partial c=\int_{\gamma(c)} d \alpha / d P$. Согласно утверждению, интеграл формы $d \alpha / d P$ по любому циклу, лежащему на поверхности $P=0$, равен 0 . Согласно теореме из $\S 6$, форма $d \alpha / d P$ имеет на «бесконечно удаленном» дивизоре $S$ проективной компактификации поверхности $P=0$ полюс не выше первого порядка. Согласно теореме 2 из $\S 5$, форма $d \alpha / d P$ тождественно равна нулю на поверхности $P=0$. Форма $d \alpha$ равна $Q d x_{1} \wedge \cdots \wedge d x_{k}$, где $Q$ - многочлен степени не выше $n-k$. Многочлен $Q$ не может делиться на $P$, так как степень многочлена $P$ равна $n$. Поэтому форма $d \alpha / d p= \pm\left(Q / P_{x_{i}}^{\prime}\right) d x_{1} \wedge \cdots \wedge d x_{i-1} \wedge d x_{i+1} \wedge \cdots \wedge d x_{k}$ обращается в нуль на поверхности $P=0$, если и только если $Q$ тождественно равен нулю или, другими словами, если и только если форма $\alpha$ точна.

\section{§8. Завершение доказательства основной теоремы}

Для завершения доказательства основной теоремы, сформулированной во введении, нам осталось проверить, что при некоторых ограничениях компактная компонента связности аффинной вещественной алгебраической гиперповерхности не гомологична нулю на проективной компактификации комплексификации этой поверхности. Здесь приводятся точная формулировка и доказательство нужного факта.

Пусть $P\left(x_{1}, \ldots, x_{k}\right)$ - вещественный морсовский многочлен степени $n$, комплексификация которого имеет лишь невырожденные критические точки с различными критическими значениями. Пусть невырожденна старшая однородная часть $P_{n}$ многочлена $P$, и пусть $P=0$ - неособая поверхность. Вешественная поверхность $P=0$ может состоять из нескольких связных многообразий, компактных или некомпактных. Эти многообразия ориентированы (как границы областей $P<0$ ). Поэтому компактные компоненты поверхности $P=0$ можно рассматривать как $(k-1)$-мерные циклы, и по ним можно интегрировать $(k-1)$-формы. 
Теорема. Если степень многочлена $P$ больше, чем $k$, то вешественнал компактная компонента $\gamma_{0}$ поверхности $P=0$ не гомологична нулю на проективной компактификачии комплексификачии этой поверхности.

ДокАЗАтЕльство. Рассмотрим форму $\alpha=x_{1} d x_{2} \wedge \cdots \wedge d x_{n}$. Или цикл $\gamma_{0}$, или цикл $-\gamma_{0}$ ограничивают компактную область $D$ в вещественном пространстве $\mathbb{R}^{k}$ (это зависит от знака многочлена $P$ в окрестности цикла $\gamma_{0}$ в области $D$ : если знак отрицателен, то цикл $\gamma_{0}$ ориентирован как $\partial D$, если положителен, - то как - $\partial D)$. Остановимся на первом случае (второй рассматривается аналогично). При малых вещественных $c$ на поверхности $P=c$ циклу $\gamma_{0}$ соответствует единственный цикл $\gamma_{c}$. Фиксируем в $\mathbb{R}^{k}$ стандартное скалярное произведение. Так как $d \alpha$ - форма объема, то из геометрических соображений видно, что при вещественных $c$

$$
\left.\left(\frac{\partial}{\partial c} \int_{\gamma_{c}} \alpha\right)\right|_{0}=\left(\int_{\gamma_{0}} \frac{d S}{\|\operatorname{grad} P\|}\right)>0,
$$

где $d S$ - элемент $(k-1)$-мерной площади поверхности и $\|\operatorname{grad} P\|-$ длина градиента многочлена $P$.

С другой стороны,

$$
\left.\left(\frac{\partial}{\partial c} \int_{\gamma_{c}} \alpha\right)\right|_{0}=\int_{\gamma_{0}} \frac{d \alpha}{d P}=\int_{\gamma_{0}} \frac{d x_{1} \wedge \cdots \wedge d x_{k}}{d P} .
$$

Согласно теореме из $\S 6$, форма $\tau=\left(d x_{1} \wedge \cdots \wedge d x_{k}\right) / d P$ при $\operatorname{deg} P>k$ голоморфна на компактификации комплексификации поверхности $P=0$. Если бы цикл $\gamma_{0}$ был гомологичен нулю на этой компактификации, то $\int_{\gamma_{0}} \tau$ равнялся бы нулю. Полученное противоречие доказывает теорему.

\section{§9. Доказательство следствия}

Докажем следствие основной теоремы, сформулированное во введении.

1. Пространство $K_{H}$ неособых компактных компонент связности линий уровня вещественного многочлена $H$ имеет структуру одномерного вещественного аналитического многообразия (вообще говоря, несвязного). Немного пошевелив, если надо, коэффициенты многочлена $H$, можно считать, что все его комплексные критические точки морсовские, все критические значения различны, а старшая однородная часть $H_{n}$ невырожденна. Определим линейное пространство $I_{n-1}$ аналитических функций на пространстве $K_{H}$. Каждая функция $f_{\alpha}$ из $I_{n-1}$ определяется формой $\alpha$ из пространства $\Omega_{n-1}$, состоящего из 1-форм, коэффищиенты которых - многочлены степени, не превосходящей $n-1$. По определению функция $f_{\alpha}$ сопоставляет кривой $\gamma$ число $\int_{\gamma} \alpha$.

2. Согласно основной теореме, пространство $I_{n-1}$ является факторпространством пространства $\Omega_{n-1}$ по пространству точных форм. Поэтому его размерность легко вычислить, а именно, она равняется $(n-1) n / 2$. Следовательно, для любого подмножества $M \subset K_{H}$, содержащего не более $(n-1) n / 2-1$ точек, существует форма $\alpha \in \Omega_{n-1}$, такая, что функция $f_{\alpha}$ не равна тождественно нулю и обращается в нуль в точках множества $M$. 
3. Из основной теоремы вытекает, что ненулевая функция из $I_{n-1}$ не равна тождественно нулю на всякой компоненте многообразия $K_{H}$. Следовательно, немного продеформировав множество $M$, можно добиться, чтобы соответствующая функция $f_{\alpha}$ имела простые нули в точках этого множества.

4. Рассмотрим малое негамильтоново возмущение $V=V_{H}+\delta j(\alpha)$ гамильтонова векторного поля $V_{H}$ с гамильтонианом $H$, где $j$ - изоморфизм кокасательного и касательного пространств, соответствующий стандартной симплектической структуре на плоскости, и $\delta$ - малое вещественное число. Согласно критерию Пуанкаре-Понтрягина [1], поле $V$ будет иметь предельные циклы вблизи кривых, соответствуюших точкам множества $M$ (на котором функщия $f_{\alpha}$ имеет некратные нули). Следствие доказано.

ЗАмЕчАнИЕ. Следствие использует основную теорему лишь в случае $k=2$. В этом случае как разложение Лефшеца, так и теоремы 1,2 из 55 почти очевидны. В этой ситуации теорема о разложении Лефшеца утверждает, что каждый одномерный цикл на компактной кривой $X$ гомологичен циклу, не пересекающему заданный дивизор $S$. Действительно, если цикл пересекает $S$, то малой деформацией цикла от этого пересечения можно избавиться. В рассматриваемом случае теорема 1 утверждает, что должна обратиться в тождественный нуль голоморфная 1-форма $\omega$ на компактной кривой $X$, интеграл от которой по всякому циклу $\gamma$, не пересекающему заданный дивизор $S$, равен нулю. Но неопределенный интеграл от формы $\omega$ в этих условиях является голоморфной Функцией на компактной кривой $X$. Значит, эта функция равна константе, и, следовательно, форма $\omega$ тождественно равна нулю. В нашей ситуации теорема 2 также очевидна - 1-форма $\omega$, имеюшая полюсы не выше 1-го порядка и нулевые вычеты, является голоморфной 1-формой. Таким образом, доказательство следствия не использует ни теоремы о разложении Лефшеца, ни теоремы Ходжа-де Рама.

\section{ЛитературА}

1. Понтрягин Л. С. О динамических системах, близких к гамильтоновым. ЖКЭТФ, 4, №8, 234-238 (1934).

2. Ильлщенко Ю. С. Пример уравнений $d w / d z=P_{n}(z, w) / Q_{n}(z, w)$, имеющих счетное число предельных циклов и сколь угодно большой жанр по Петровскому-Ландису. Матем. сб., 80, №3, 388-404 (1969).

3. Jesus Mucino-Raymundo. Deformations of holomorphic foliations having a meromorphic first integral. J. Reine Angew. Math., 461 (95), 189-219.

4. Арнольд В. И., Варченко А. Н., Гусейн-Заде С. М. Особенности дифференцируемых отображений. Т. 2. Наука, М. (1984).

5. Южаков А. П. Методы вычисления многомерных вычетов. Совр. проблемы математики. Фундаментальные направления. Итоги науки и техники. Т. 8, ВИНИТИ, М. (1985), с. 7-10.

6. Шафаревич И. Р. Основы алгебраической геометрии. Наука, М. (1972).

Московский государственный университет им. М. В. Ломоносова, механико-математический факультет Московский независимый университет
Поступило в редакцию

16 сентября 1996 г.

В переработанном виде 24 декабря 1996 г. 